\title{
Performance of Rocketdyne Phase-optimized Pure Permanent Magnet Undulator,
}

\author{
G. Rakowsky, B. Bobbs, J. Brown, P. Kennedy and G. Swoyer \\ Rockwell International, Rocketdyne Division \\ 6633 Canoga Avenue, Canoga Park, CA 91303
}

\section{Abstract}

Preliminary results of magnetic measurements of a high field, pure permanent magnet (PPM) undulator are presented. A two-step optimization process, using simulated annealing, was applied to reduce the RMS field error and to minimize RMS phase shake, trajectory walkoff and integrated dipole and multipole field errors by optimally arranging the magnets. The field-terminating end module design is a novel implementation of Halbach's displacement-free termination scheme. Simulations of entry/exit trajectorics for this termination are compared to trajectories computed from field measurements.

\section{INTRODUCTION}

A high-field, pure permanent magnet (PPM) undulator design [1] has been under development at Rocketdyne with the objective of delivering optimal performance in free electron lasers (FEL's) and in synchrotron radiation (SR) sources. The iron-free structure allows construction of field models by superposition of measured fields of individual magnets or modules, and permits evaluation of the performance of any given arrangement of magnets. An optimization algorithm such as simulated annealing [2] may then be used to select and sort magnets or modules to minimize various errors and to optimize device performance. The effectiveness of SA in minimizing trajectory wander has been demonstrated in Rocketdyne's Wiggler I, [3] an 80-period PPM device with a low field, "classical" 4-block-per-period geometry. Wiggler II was constructed to implement the high field, 6-block design and to demonstrate the higher level of optimization possible with this structure. This paper reviews the design of Wiggler II and discusses the results obtained from initial field mappings

The last section describes the novel, non-steering, displacement-frec end sections installed on Wiggler II. Entry/exit trajectories computed from the field maps are compared with simulations.

\section{WIGGLER II DESIGN}

The basic design, shown in Figure 1, is an implementation of the Halbach PPM wiggler concept [4] and has been described previously. It employs 6 magnets per period in two opposing arrays forming a planar wiggler or undulator. The magnetic structure uses two basic magnet types: Type A with vertical magnetization and Type B magnetized at $60^{\circ}$ from the vertical. Magnet dimensions have been optimized to achieve $93 \%$ of the theoretical maximum on-axis field obtainable in a planar Halbach PPM structure.

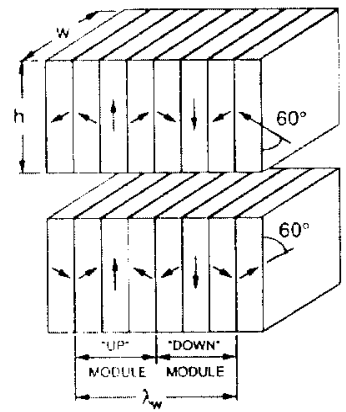

Figure 1. High ficld PPM structure.

Each half-period triplet module consists of an A magnet flanked by two B magnets, clamped in an aluminum holder. There are two types of modules, "up" and "down", which alternate to form the periodic structure (Figure 1). The magnet holders are located by dowel pins in the I-beam-supported aluminum magnet trays. Independent stepper motor drives allow tapering of the magnetic gap in FEL's. The mechanical design is shown in Figure 2. Table 1 lists the key parameters.
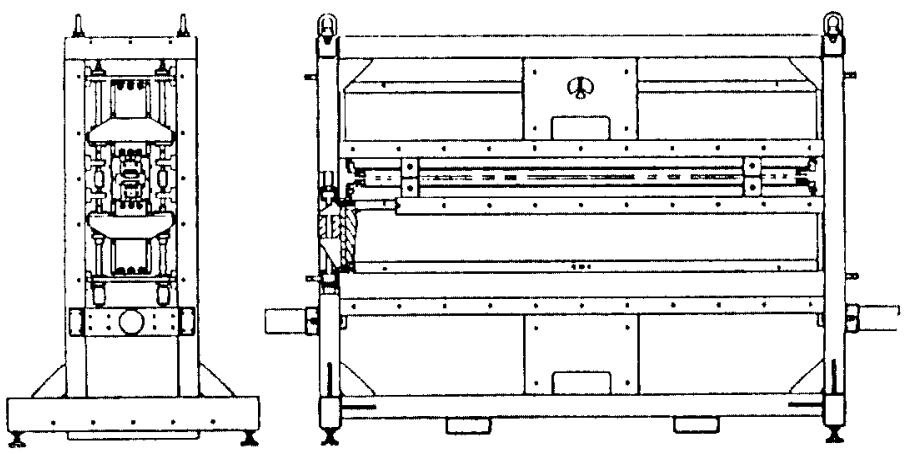

Figure 2. Wiggler II mechanical design.

Table 1

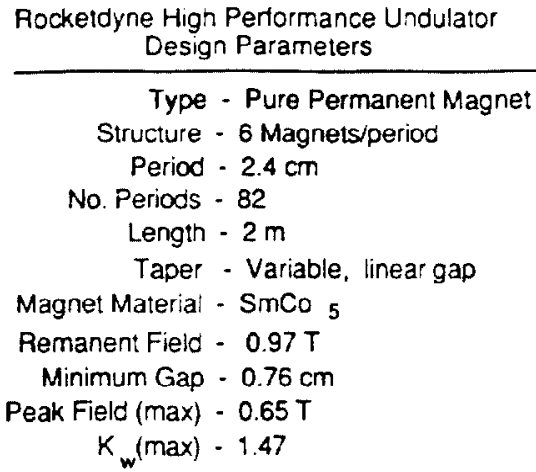


The $2 \mathrm{~m}$ long trays were designed for an 83-period structure plus half-strength end modules as well as rotatable trim magnets. However, the structure was shortened to 78 periods to accomodate the new, displacement-free terminations, and to permit adequate mapping of the entry/exit regions by the externally-mounted, dual Hall probe field mapper, whithin the travel limits imposed by the wiggler frame.

\section{OPTIMIZATION}

The natural modularity and symmetry of the 6-block geometry lends itself to a two-step optimization process. A total of 1245 magnets (415 Type A and 830 Type B) were purchased and individually characterized in each orientation, using the Hall probe scanning technique. Effects of ambient temperature and probe drift were accounted for by scanning a reference magnet along with the magnet under test. This resulted in a repeatability error of $0.04 \%$. The integrated field "kick" values, were input to a "Stage 1" version of the SA code which selected and arranged magnets to form an optimized set of 190 "up" and 190 "down" triplets with minimized variation in triplet strength. The modules were assembled as prescribed and recharacterized. With magnet tolerances of $\pm 2 \%$ on remanence and $\pm 1^{\circ}$ on magnetization angle, the code predicted a variation of triplet strength of $\sigma=1.25 \%$ for random selection of magnets, while for the annealed set the prediction was $\sigma=0.19 \%$. The actual measured variation was $\sigma=0.23 \%$.

For the next stage of optimization, the 380 triplet modules were remapped for both $x$ and $y$ field components, on- and offaxis. The on-axis By scan data were used to set up the modificd Kincaid-typc, quasi-sinusoidal ficld and trajectory models described by Bobbs et al.[5] This model takes into account the field overlap among neighboring modules and distributes the errors associated with each triplet among neighboring intervals. The transverse ( $\mathrm{Bx}$ ) field components were modeled as a sequence of point-kicks, and the out-ofplane $(y-z)$ trajectory was then represented by a simpler, piecewise-linear model.

The algorithm for Stage 2 optimization selects and arranges triplet modules with appropriate orientation in the wiggler array, then evaluates the RMS walkoff and phase shake in $x$ and $y$ from the models desribed above. In addition, the on-axis and off-axis field integrals of the individual triplets in the array are summed to compute the predicted normal and skew dipole, quadrupole and sextupole errors for the given configuration. The total "cost function" is a weighted sum of these 10 error terms, which is evaluated for each iteration. The SA algorithm provides a systematic search for an arrangement which produces a global minimum of the cost function.

\section{Preliminary Results}

Initial field maps have been taken of the 78-period structure for both the normal $(\mathrm{By})$ and transverse $(\mathrm{Bx})$ field components, on and off-axis. The structure includes the displacement-free terminations, discussed in the next section. Data were taken at a gap of $11 \mathrm{~mm}$. Analysis shows that the measured peak field of 0.42 Tesla agrees with the value predicted by the Halbach formula to better than $1 \%$. As expected, the field of the 6block geometry has very low harmonic content, with only $0.25 \%$ measured third harmonic. The RMS ficld errors are $0.83 \%$ for By and $0.34 \%$ for Bx. Comparison of the By field error with the $0.23 \%$ RMS variation of triplet strength found earlier, suggests that the bulk of the error probably comes from construction errors.

Integrating the fields twice and scaling to an electron gamma of 80 yields the trajectory in the $\mathrm{x}-\mathrm{z}$ wiggle plane shown in Figure 3. In the calculation we subtracted a $D C$ field offset of 0.25 Gauss. The RMS walkoff in the $\mathrm{x}-\mathrm{z}$ plane is 0.32 of the wiggle ampltude, well within target and similar to that obtained in Wiggler I . Walkoff (Xcrr), which is computed as the difference between the trajectory and a best-fit sinusoid over the core of the undulator, is also plotted in Figure 3.

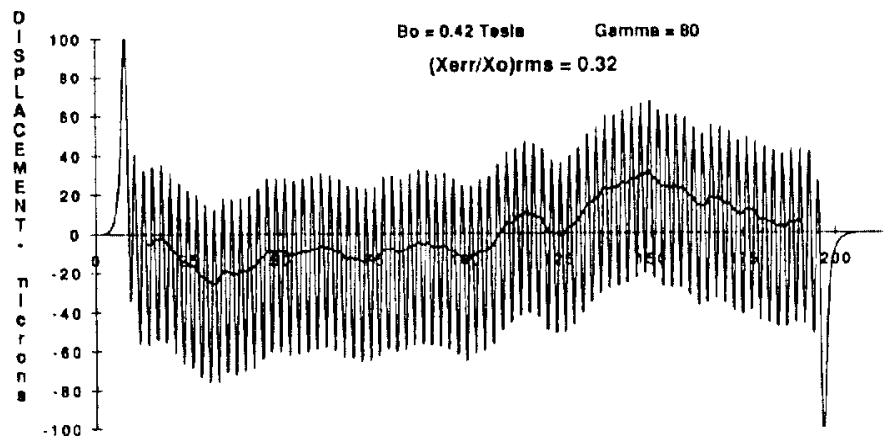

Figure 3. Trajectory in wiggle plane and walkoff error.

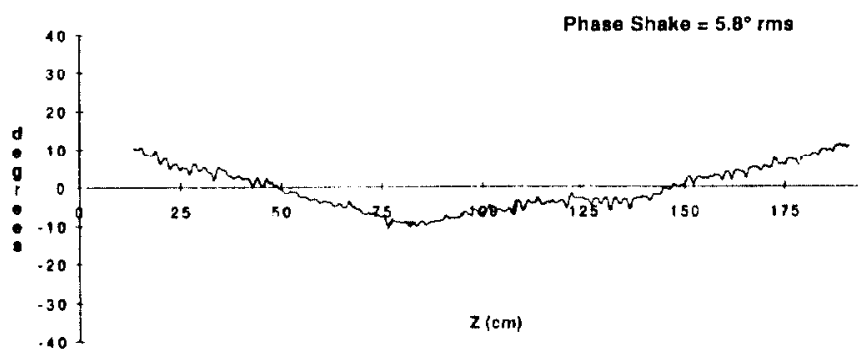

Figure 4. Phase error in $x-z$ plane.

Phase error was computed by numerically integrating the path lengths along both the wiggle trajectory and the best-fit sinusoid and expessing the difference in degrees of phase at the resonant optical wavelength. A plot of the phase error is shown in Figure 4. Overall, the RMS value of the phase error (phase shake) is $5.8^{\circ}$. According to statistical studies by Kennedy et al.,[6] this should assure small-signal FEL gain or fundamental spectral brightness of better than $90 \%$ of ideal. However, the gross feature of the plot is a bow, indicating a long-range, systematic field variation, which may be removed by adjusting the gap taper. The remaining phase variations duc to local magnet errors are then quite small, on the order of 1 $2^{\circ}$, as expected.

Calculation of the $y-z$ trajectory form the $B x$ field data showed a severe walkoff, indicating that perhaps the Bx field errors were accumulating instead of cancelling. Indeed, it was found that the formulation of the $y-z$ walkoff erroncously 
included an alternating negative sign. One could correct this error by simply rotating every other module $180^{\circ}$ about the vertical axis. However, such a reversal would also affect the quadrupole cost function, which is sensitive to module orientation. It appears then, that the best solution would be to disassemble the arrays and redo Stage 2 annealing.

Another source of error was the fact that the trays were fabricated separately from the support beams, resulting in peak gap variations of up to 40 microns and a twist of about $1^{\circ}$. We attempted to compensate for the gap errors by introducing a field scaling correction factor in the formulation of the cost function. From the plot of trajectory error it appears that the compensation was only partially successful. Clearly, the better approach would be to eliminate the problem by regrinding the trays mounted on their support beams.

Quadrupole and sextupole errors were not minimized adequately either. Again, this may be due in large part to the gap errors.

\section{END SECTION DESIGN}

Two different termination schemes have been considered for Wiggler II. The first was a non-steering termination using half-strength end modules. In the 6-block geometry such a module is easily realized with just a single Type A magnet flanked with nonmagnetic "dummy" blocks. The arrangement is illustrated in Figure 5a. The magnets could be selected to cancel any residual trajectory steering. Since the terminating magnets are the same size and type, and are at the same gap as the core of the structure, their fields should track with gap changes and the trajectory should remain true. This scheme does, however, produce a small trajectory displacement, on the order of a wiggle amplitude.

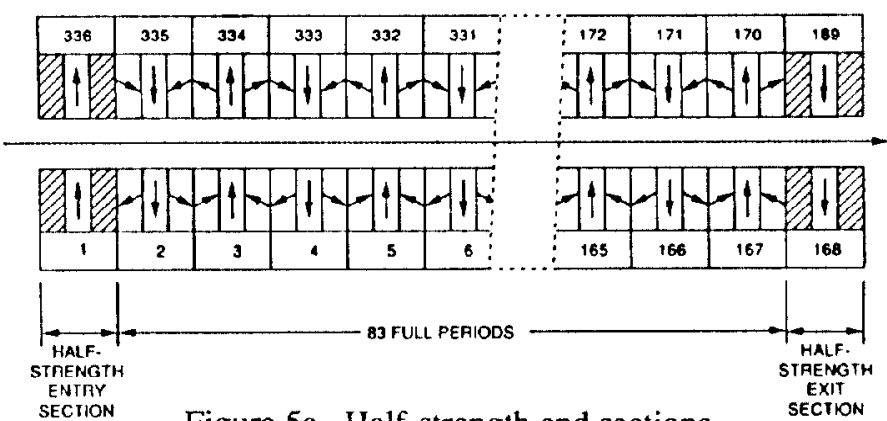

Figure 5a. Half-strength end sections.

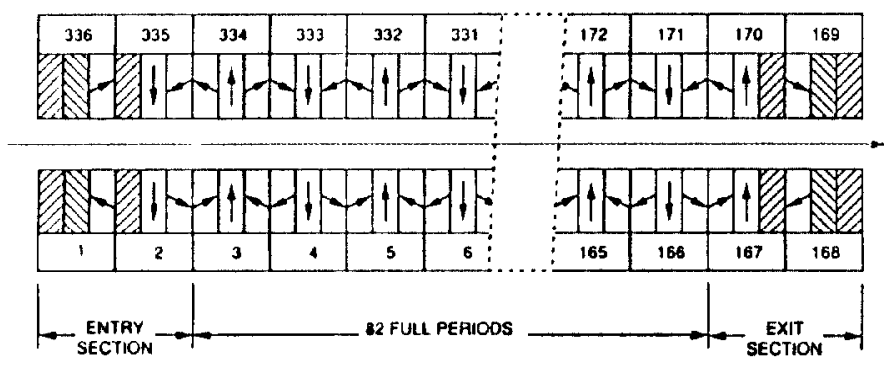

Figure 5b. Non-steering, dispplacement-free termination.
Recently Klaus Halbach suggested a displacement-free, non-steering termination scheme for PPM structures analogous to the arrangement he devised for hybrids.[7] We developed a variant of the scheme which again uses only standard magnets and modules, with one or two magnets replaced by dummy blocks. This termination, shown in Figure $5 \mathrm{~b}$, has been implemented in Wiggler II.

To simulate the trajectory through the new end section, field scan data of a typical A-type and B-type magnet were used to construct by superposition the fields in the entry region. These were then twice integrated numerically to obtain the plots shown in Figures 6 . The form of the trajectory shown in Figure 3 agrees well with the simulation. The 12 magnets used in the terminations were initially chosen at random to confirm the validity of the termination design. Final selection will be done by a Stage 3 optimization process, to cancel any remaining trajectory or multipole errors.

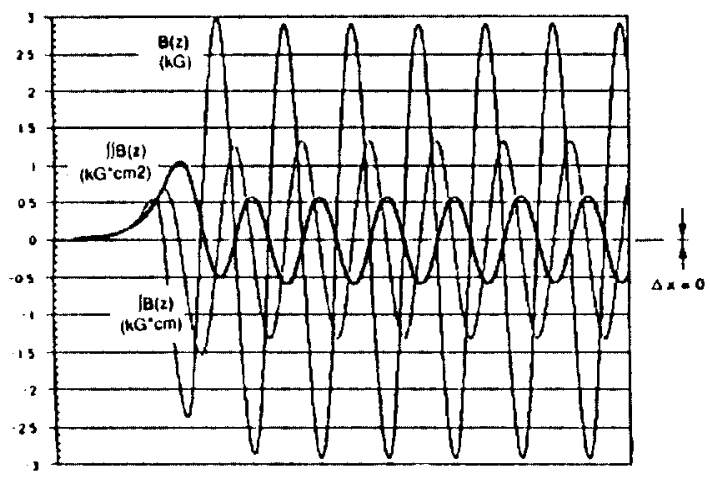

Figure 6. Simulation of fields and trajectories through entry region with displacement-free termination.

\section{ACKNOWLEDGEMENT}

The authors thank Cynthia Todd and Youssef Kohanzadeh for their assistance in hardware assembly, mapping and data reduction

\section{REFERENCES}

[1] G. Rakowsky et al., "High Performance Pure Permanent Magnet Undulators," Proc. 11th Int'l. FEL Conf., Nucl. Inst. Meth. ,vol. A296, pp. 597-602, 1990.

[2] A. Cox and B. Youngman, "Systematic Selection of Undulator Magnets Using the Technique of Simulated Annealing," Proc. SPIE, vol. 582, p.91, 1986.

[3] M. Curtin et.al., "A High Quality Permanent-Magnet Wiggler for the Rocketdyne/Stanford Infrared Free Electron Laser", Proc. IX Int'l. Free Electron Laser Conf., Nucl. Inst. Meth. vol. A272, pp. 187-191, 1988.

[4] K. Halbach, "Physical and Optical Properties of Rare Earth Cobalt Magnets," Nucl. Inst. \& Meth., vol. 187, pp. 109. 117,1981

[5] B. Bobbs et al., "In Search of a Meaningful Field Error Spec for Wigglers", Proc. 11th Int'l. FEL Conf., Nucl. Inst. Meth. ,vol. A296, pp. 574-578, 1990.

[6] P. Kennedy et al., "Statistical Variation of FEL Performance Due to Wiggler Field Errors," Proc. 11th In't. FEL Conf., Nucl. Inst. Meth. ,vol. A296, pp. 607-610, 1990.

[7] Klaus Halbach, private communication, August 24, 1990. 